\title{
Étude des caractéristiques chimiques et microbiologiques des ressources en eau du bassin versant du N'zi : cas de la commune de N'zianouan (Sud de la Côte d'Ivoire)
}

\author{
Kouassi Ernest AHOUSSI ${ }^{1 *}$, Yao Blaise KOFFI ${ }^{1}$, Amani Michel KOUASSI ${ }^{2}$, \\ Gbombélé SORO ${ }^{1}$, Nagnin SORO ${ }^{1}$ et Jean BIEMI $^{1}$ \\ ${ }^{1}$ Laboratoire des Sciences et Techniques de l'Eau et de l'Environnement (LSTEE), Unité de Formation et de \\ Recherche (UFR) des Sciences de la Terre et des Ressources Minières (STRM) Université de Cocody, \\ 22 BP 582 Abidjan 22 ; Tel : (+225) 224838 03, Côte d'Ivoire. \\ ${ }^{2}$ Département des Sciences de la Terre et des Ressources Minières (STeRMi), Institut National Polytechnique \\ Félix Houphouët Boigny (INP HB), BP 1093 Yamoussoukro, Côte d'Ivoire, Tél. (225) 30646715 ; \\ *Auteur correspondant, E-mail: ahoussi@gmx.fr
}

\section{RESUME}

Cette étude traite de la caractérisation chimique et microbiologique des eaux de la commune N'zianouan. Cette commune, dépourvue d'un système d'assainissement adéquat et correctement fonctionnel est confrontée à un problème de protection de ses ressources en eaux. C'est pour mettre en évidence l'influence de la pression anthropique sur les eaux de la rivière N'zi aux droits de cette localité et également sur les eaux souterraines que cette étude a été initiée. La méthode utilisée pour atteindre cet objectif repose sur des mesures de niveaux piézométriques de la nappe souterraine, des analyses physico-chimiques et microbiologiques des eaux. Les résultats de ces analyses ont été traités à l'aide de la classification hydrochimique basée sur le diagramme de Piper et de l'analyse statistique multivariée. La méthode statistique a fait intervenir la Classification Hiérarchique Ascendante (CHA) pour l'identification des phénomènes responsables de la minéralisation des eaux de la région. L'étude piézométrique montre que le niveau piézométrique de la nappe varie de $0,48 \mathrm{~m}$ à $4,52 \mathrm{~m}$, avec une moyenne de $1,24 \mathrm{~m}$. La température des eaux varie de $27,6{ }^{\circ} \mathrm{C}$ à $30^{\circ} \mathrm{C}$, avec une moyenne de $28,48^{\circ} \mathrm{C}$. La forte valeur de conductivité a été observée au niveau des eaux souterraines $(2487 \mu \mathrm{S} / \mathrm{cm})$. Les plus forts taux de matières en suspension sont observés dans les eaux de la rivière N'zi (16 $\mathrm{mg} / \mathrm{L}$ ). Elles présentent une bonne saturation en oxygène dissous. Les eaux étudiées se classent en deux grands groupes. Il s'agit des eaux bicarbonatées calciques pour les eaux de la rivière N'zi et des eaux chlorurées sodipotassiques pour les eaux souterraines. Dans ces eaux, sont également présentes des teneurs élevées en Eléments Traces Métalliques (ETM) tels que : l'arsenic, le plomb, le chrome et le cuivre. Sur le plan microbiologique, ces eaux contiennent un fort taux de coliformes fécaux principalement l'Echérichia coli (15000 ; 12200 ; et 14500 Echérichia coli pour $100 \mathrm{ml}$ d'eau). Cette étude signale également la présence de streptocoques fécaux, de Clostridiums Perfringens et de coliformes Thermo tolérants dans les ressources en eau. Les coliformes Thermo tolérants sont les plus importants dans le milieu, avec des quantités de 24600 et de 112700 pour les eaux de la zone. Les teneurs sont plus importants au niveau des eaux souterraines (112700 coliformes Thermo tolérants/100ml) qu'au niveau des eaux de surface. L'ensemble des résultats obtenus montre que les ressources en eau de N'zianouan sont généralement soumises à une pression anthropique importante.

Mot clés: Classification Hiérarchique Ascendante, diagramme de Piper, hydrochimie, environnement, Eléments Traces Métalliques. 


\section{INTRODUCTION}

Depuis ces dernières décennies, les études réalisées sur les ressources en eau de Côte d'Ivoire par les auteurs tels qu'Ahoussi et al. (2008, 2009, 2010 et 2011), Soro et al. (2010) ont signalé de nombreux foyers de pollution aussi bien au niveau des eaux de surface que des eaux souterraines, dans les localités telles qu'Abidjan dans les communes de Koumassi, Marcory et Adjamé. Ces études ont également montré que la lagune Ebrié qui borde la ville d'Abidjan est polluée par les activités domestiques et industrielles de la ville (Coulibaly et al., 2010). En effet, la croissance démographique que connait le pays après l'indépendance a eu pour conséquence l'augmentation des besoins primaires des populations dont l'accès à l'eau potable, aux denrées alimentaires, à la santé et éducation en générale. Cette grande pression démographique sur les ressources naturelles se traduit par une réduction des terres agricoles et la production de plus en plus importante de déchets de toute nature. A cela s'ajoute le manque d'un système d'assainissement adéquat dans le pays. Ainsi dans les grandes villes telles qu'Abidjan, les déchets domestiques se retrouvent dans la nature ou dans les cours d'eau. Les eaux de surface qui sont plus vulnérables sont devenues aujourd'hui de véritables sites de vidanges des eaux usées domestiques et des eaux vannes. Ces cours d'eau sont également devenus des collecteurs de déchets et d'ordures de toutes natures. Les eaux des nappes phréatiques sont également soumises à cette pression anthropique qui participe à la dégradation de leur qualité.

La commune de N'zianouan, situé dans le département de Tiassalé n'est pas en marge de ces problèmes environnementaux. Ladite localité objet de cette étude est défavorisée par l'inexistence d'un système d'assainissement adéquat et on y observe la présence de tas d'ordures à plusieurs endroits. Les effluents de cette localité sont généralement déversement dans la rivière N'zi; car, elle est située dans le bassin versant de ce cours d'eau.

Cette rivière est donc le réceptacle principal de la totalité des eaux usées de la ville. Elle est également devenue un collecteur de déchets domestiques et agricole de toutes sortes. C'est pour évaluer l'influence des activités anthropiques sur la qualité des ressources en eau de la commune de N'zianouan que cette étude a été initié. Elle s'appuie sur l'utilisation des données piézométriques, hydrochimiques et microbiologiques pour la caractérisation de la pollution des eaux et des phénomènes responsables de la dégradation de la qualité des eaux de cette commune.

\section{MATÉRIEL ET MÉTHODES \\ Présentation de la zone d'étude Géographie et géomorphologie}

La commune de N'zianouan est située dans le département de Tiassalé sur l'autoroute du Nord dans le bassin versant de la rivière N'zi (Figure 1). Cette localité objet de cette étude est située à $30 \mathrm{~km}$ de la ville de Tiassalé et à $100 \mathrm{~km}$ d'Abidjan. Elle abrite une population estimée en 1998 à 7427 habitants composée de 4086 Hommes et 3 341 Femmes. On y rencontre des populations autochtones (les baoulés) et des ressortissants de la sous région (Burkinabés, Nigérians, Maliens, etc.).

Le relief de la région généralement plat est constitué en grande partie de plaines. Dans cette zone, on y rencontre également des collines (108 m d'altitude en moyenne) et des vallées. En outre, les massifs intrusifs de Binao et de Boussoukro offrent une morphologie particulière caractérisé par l'existence d'inselbergs et de chaos granitiques notamment entre les villes de Tiassalé et N'Douci. Cette zone bénéficie d'un climat de type tropical humide, localement appelée climat «attiéen». Les pluies y sont abondantes et se repartissent en deux saisons séparées par deux saisons sèches et deux saisons pluvieuses. La grande saison sèche débute de décembre à mars et 
est marquée par 1'harmattan. La grande saison des pluies commence d'avril à juillet, avec le mois de mai qui est le plus pluvieux. Une petite saison sèche d'août à septembre et une petite saison des pluies d'octobre à novembre sont également observables.

En ce qui concerne les températures moyennes mensuelles, elles varient de 25 à 29 ${ }^{\circ} \mathrm{C}$. La période la plus chaude de l'année est celle de janvier à avril, avec une température maximale de l'ordre de $29{ }^{\circ} \mathrm{C}$ en mars. L'insolation quant à telle, varie de 3 à 7,4 h/j sur l'ensemble de la zone d'étude et dure en moyenne $6 \mathrm{~h} / \mathrm{j}$.

Le cours principal de N'zianouan est la rivière N'zi, affluent du Bandama et qui prend sa source au Nord de la Côte d'Ivoire dans la région de Ferkéssedougou à une altitude de $400 \mathrm{~m}$. Il coule globalement dans une direction Nord-Sud (Figure 2).

Le débit de la rivière N'zi mesuré à la station limnimétrique de N'zianouan est donné par la Figure 3. Les crues les plus importantes sont enregistrées à N'zianouan au cours du mois d'octobre où on a des débits mensuels moyens extrêmes de $320 \mathrm{~m}^{3} / \mathrm{s}$.

Géologie et hydrogéologie de la zone d'étude

La géologie de la zone d'étude se confond avec celle de la région de Tiassalé. Les formations géologiques ont été structurées au cours de l'orogenèse éburnéenne. Elles sont formées d'ensembles volcanosédimentaires constituées de schisteuses et de méta-arénites (Delor et al., 1992), dans lesquels apparaissent en intrusion des volcanites et des granitoïdes éburnéens (granites à biotites et granodiorites), avec des auréoles de métamorphisme (Beliard et Casanova, 1972) (Figure 4).

Les massifs intrusifs sont nombreux et divers dans la région. Les plus significatifs sont des intrusions de granites (à deux micas, à muscovite), de granodiorites et de tonalites. Ces intrusions sont d'étendue variable dans les schistes birimiens et sont masquées par les altérites et/ou la végétation. Les granites à biotite affleurent au Sud-Ouest de N'douci. Ils renferment des enclaves mafiques à grains fins et des enclaves ultramafiques qui sont microgrenues. Les Granites à muscovite se rencontrent à N'Douci et à Niamazra à l'Est de Tiassalé. Ces granites sont très répandus à la sortie de N'Douci dans le sens de Tiassalé où on les retrouve sous forme de chaos granitique (Figure 5).

Les granodiorites sont présents dans la localité de Boussoukro. Les monzonites affleurent dans les environs de la ville de Tiassalé, et dans le triangle Morokro-AhiroaAttinguié. Ce sont également des roches à grains moyens et grossiers.

Les tonalites apparaissent dans la région de Boussoukro. Les monzosyénites sont limités cartographiquement à un massif annulaire affleurant au Sud-Ouest de N'Douci et à une intrusion kilométrique incluse dans la tonalite de Boussoukro (Beliard et Casanova, 1972).

Sur le plan structural, l'ensemble de la région est parsemée de fractures dont la plupart sont remplies par des filons de quartz parfois broyés. La distribution des fractures et des fissures n'épouse pas celle des grands sillons birimiens orientés NNE-SSW (Tagini, 1971). Ces fractures sont de grandes extensions puisqu'on les retrouve dans le bassin de la Mé situé à l'Est de la région d'étude (Tapsoba, 1995). Les amphibolites de la ceinture volcanique qui se trouvent dans le lit de la rivière $\mathrm{N}^{\prime} z i$ à Tiassalé ont une orientation $\mathrm{N} 25^{\circ}$ et $\mathrm{N} 50^{\circ}$.

\section{Cadre hydrogéologique}

Du point de vue hydrogéologique, deux types d'aquifères sont présents dans la région. Il s'agit des aquifères d'altérites et des aquifères de fissures. Les aquifères d'altérites se développent dans les couches d'altération plus ou moins épaisses. Ces altérites présentent une épaisseur qui varie de $1,95 \mathrm{~m}$ à $53 \mathrm{~m}$ et sont formés d'argiles et d'arènes.

Ces aquifères reçoivent directement les eaux des précipitations et leurs niveaux piézomètriques baissent considérablement en saison sèche et remontent en saison de pluie (Biémi, 1992). Ces nappes d'altérites sont 
captées par les nombreux puits villageois de la commune de N'zianouan (Figure 6).

Les aquifères de fractures se développent dans la zone broyée et/ou fissurée. Ces aquifères occupent une place de choix dans la prospection des ressources en eau dans les régions de socle cristallin ou cristallophyllien. Souvent les aquifères d'altérites et de fissures sont hydrauliquement liés ; ce qui permet aux aquifères de fissures plus profondes de se recharger à travers les aquifères d'alterites plus superficiels. Dans la région, de nombreux forages captent ces aquifères à des profondeurs variables. Selon Djaa (2010), dans la zone, la profondeur moyenne des forages est de $55 \mathrm{~m}$ pour les granites et de $60 \mathrm{~m}$ pour les schistes. Les transmissivités des aquifères de la région de Tiassalé oscillent entre $3.10^{-6} \mathrm{~m}^{2} . \mathrm{s}^{-1}$ et $1,4.10^{-3}$ $\mathrm{m}^{2} \cdot \mathrm{s}^{-1}$, pour les schistes et entre $7,54 \cdot 10^{-5} \mathrm{~m}^{2} . \mathrm{s}^{-}$ ${ }^{1}$ et $2,85 \cdot 10^{-3} \mathrm{~m}^{2} \cdot \mathrm{s}^{-1}$ pour les granites.

\section{Méthode d'échantillonnage}

Une campagne d'échantillonnage a été effectuée en novembre 2010 dans la commune de N'zianouan. Elle a été réalisée pendant la petite saison sèche et a porté sur les eaux souterraines captées par les puits et forages et les eaux de surface (rivière N'zi). Lors de cette campagne, les échantillons d'eau prélevés ont été mis dans des bouteilles en polyéthylène de capacité 1 litre, préalablement lavées à l'acide nitrique puis à l'eau distillée. Sur le terrain, avant le remplissage des bouteilles, celles-ci ont été lavées trois fois avec l'eau à prélever. Le remplissage des bouteilles a été fait à ras bord puis le bouchon vissé afin d'éviter tout échange gazeux avec l'atmosphère. Les échantillons d'eau ont été ensuite transportés dans une glacière à $4{ }^{\circ} \mathrm{C}$ au laboratoire pour analyse dans l'heure qui suit le prélèvement. Lors des prélèvements, les paramètres physiques des eaux tels que la température, le $\mathrm{pH}$, la conductivité électrique (CE), la salinité, le taux d'oxygène dissous $\left(\mathrm{O}_{2}\right)$ et le potentiel d'oxydo-réduction ont été mesurés in situ dans les puits et forages pour les eaux souterraines et dans la rivière N'zi.
L'appareillage utilisé sur le terrain (Figure 7) est constitué d'un pH-mètre Star 4 pour la mesure du $\mathrm{pH}$ et Eh, d'un conductimètre Hach Sension 5 pour la mesure de la conductivité électrique (CE), de la température, de la salinité et les solides totaux dissous (TDS).

Au total, cinq échantillons d'eau ont été prélevés dont deux pour les eaux souterraines et trois pour les eaux de la rivière N'zi. Les éléments à doser sont constitués de paramètres chimiques et microbiologiques des eaux. Les paramètres chimiques analysés sont $\mathrm{Ca}^{2+}$, $\mathrm{Mg}^{2+}, \mathrm{Na}^{+}, \mathrm{K}^{+}, \mathrm{Cl}^{-}, \mathrm{SO}_{4}{ }^{2-}$ et $\mathrm{HCO}_{3}{ }^{-}$. Les sels nutritifs dosés sont constitués de $\mathrm{NO}_{3}{ }^{2-}, \mathrm{NO}_{2}{ }^{-}$, $\mathrm{NH}_{4}{ }^{+}$, et le $\mathrm{PO}_{4}{ }^{3-}$. Pour voir le degré de pollution métallique des eaux de la zone, les Eléments Traces Métalliques (ETM) tels que $\mathrm{Cu}, \mathrm{Pb}, \mathrm{Cr}$, et As ont été dosés. $\mathrm{La} \mathrm{DBO}_{5}$ et la $\mathrm{DCO}_{5}$ des eaux ont été également mesurées. En ce qui concerne les analyses microbiologiques, les bactéries telles que Escherichia coli, streptocoques fécaux, Clostridiums Perfringens et Coliformes Thermo tolérants ont été dosés. Ainsi, cinq échantillons d'eau ont été également prélevés dans les puits, forages et dans la rivière N'zi. L'échantillonnage des eaux de la rivière N'zi a été principalement réalisé en amont et en aval de la rivière afin que l'échantillon amont serve de témoin pour le suivi de la qualité physico-chimique et microbiologique des eaux de surface.

\section{Méthode d'étude piézométrique}

La campagne de relevés piézométriques a été réalisée en octobre 2010, ce qui correspond à la période des hautes eaux dans la région de N'zianouan. Elle a permis de déterminer le niveau piézométrique de la nappe phréatique. Les coordonnées des points d'eau ont été déterminées à l'aide d'un GPS Garmin Map 60 CSX. Les mesures piézométriques ont été réalisées à l'aide d'une sonde piézométrique OTT $(100 \mathrm{~m})$ sonore et lumineuse. Les données collectées sur le terrain ont permis de déterminer la côte des eaux souterraines selon la formule suivante : 
$\mathbf{C}_{\text {eau }}=\mathbf{Z}-\mathbf{H}$; avec $\mathrm{Z}$ : altitude du piézomètre $(\mathrm{m}) ; \mathrm{H}=(\mathrm{NP}+\mathrm{M})$ : profondeur de l'eau dans le piézomètre $(\mathrm{m})$; NP : niveau piézométrique $(\mathrm{m}) ; \mathrm{M}$ : margelle; $\mathrm{C}_{\text {eau }}$ : côte de l'eau (m).

Pendant la campagne piézométrique, la profondeur des puits a également été mesurée. La lame d'eau dans le puits a été déterminée par la formule suivante :

$\mathbf{L}_{\text {eau }}=$ Prof (puits) $-\mathbf{N P}$; avec $\mathrm{L}_{\mathrm{eau}}$ : lame d'eau en $(\mathrm{m})$; Prof (puits) : profondeur du puits en $(\mathrm{m})$;

\section{Méthode d'analyse et de traitement des données}

Au laboratoire, les cations majeurs, ont été dosés par la méthode de spectrophotométrie à l'aide d'un spectrophotomètre de type AAS VARIAN. Les sels nutritifs ont été dosées par la méthode de spectrophotométrie à partir d'un spectrophotomètre de type UV SHIMADZU. Les bicarbonates ont été dosés à partir de la méthode de dosage à l'aide d'un titrateur digital. Les chlorures ont été mesurés par la méthode de Mohr par titrage. Les sulfates, la DCO ont été dosés par spectrophotométrie à l'aide d'un spectrophotomètre HACH DR 2010. La DBO5 a été dosé par incubation à partir d'un incubateur de type HACH. La turbidité des eaux a été mesurée à partir d'un turbidimètre 2020. Les métaux ont été dosés à partir de la méthode de spectrométrie à l'aide d'un spectrophotomètre à absorption atomique VARIAN. Les analyses microbiologiques ont nécessités l'utilisation de méthodes selon les microorganismes recherchés. Ainsi, pour le dénombrement des coliformes totaux nous avons fait appel à la méthode NF V08-50 et pour le dénombrement des coliformes fécaux de type Eschérichia, la méthode AFNOR BRD 07/1-07/93 a été utilisée.

Les données collectées sur le terrain et au laboratoire ont été traitées en utilisant une combinaison de méthodes statistique mutlivariée et de méthode hydrochimique. La méthode hydrochimique à nécessité l'utilisation du diagramme de Piper pour la classification hydrochimique des eaux. En effet, ce diagramme est très fréquemment utilisé dans le domaine de hydrochimie, avec de très bon résultats (Yermani et al., 2003 ; Kouzana et al., 2007; Oga et al., 2009; Ahoussi et al., 2010; Kouassi et al., 2010). L'approche statistique est basée sur l'utilisation de la Classification Hiérarchique Ascendante (CHA) pour l'étude des phénomènes à l'origine de la minéralisation des eaux. La Classification Hiérarchique Ascendante (CHA) est un outil puissant pour analyser des données de la chimie d'eau et pour la formulation de modèles géochimiques (Meng et Maynard, 2001 in Yidana, 2007). C'est un système de classification qui utilise la distance euclidienne pour des mesures de similitude et la méthode de la tutelle pour lien qui produit la classification la plus distinctive où chaque membre dans un groupe est plus semblable à ses collègues qu'à n'importe quel membre à l'extérieur du groupe (Güler et al., 2002). La CHA a été réalisée à partir de 5 descripteurs et 23 variables qui sont : minéralisation totale (MT), $\mathrm{pH}, \mathrm{HCO}_{3}^{-}, \mathrm{SO}_{4}{ }^{2-}$, Turb, Eh, $\mathrm{O}_{2}, \mathrm{~T}^{\circ}, \mathrm{CE}, \mathrm{MES}, \mathrm{Sal}, \mathrm{Cl}^{-}, \mathrm{Na}^{+}, \mathrm{K}^{+}$, $\mathrm{Ca}^{2+}, \mathrm{Mg}^{2+}, \mathrm{NO}_{3}^{-}, \mathrm{NO}_{2}{ }^{-}, \mathrm{NH}_{4}{ }^{+}, \mathrm{As}, \mathrm{Cu}, \mathrm{Cr}$ et $\mathrm{Pb}$. Les analyses statistiques ont été réalisées à l'aide du logiciel STATISTICA 6.0. Les résultats des analyses microbiologiques ont été comparés aux valeurs guides de l'Organisation Mondiale de Santé (OMS) dans le cadre d'une eau de boisson, non influencée par les activités anthropiques. Toutes les différentes méthodes utilisées dans cette étude ont permis de connaitre l'influence des activités anthropiques sur les ressources en eau de la commune de N'zianouan. 


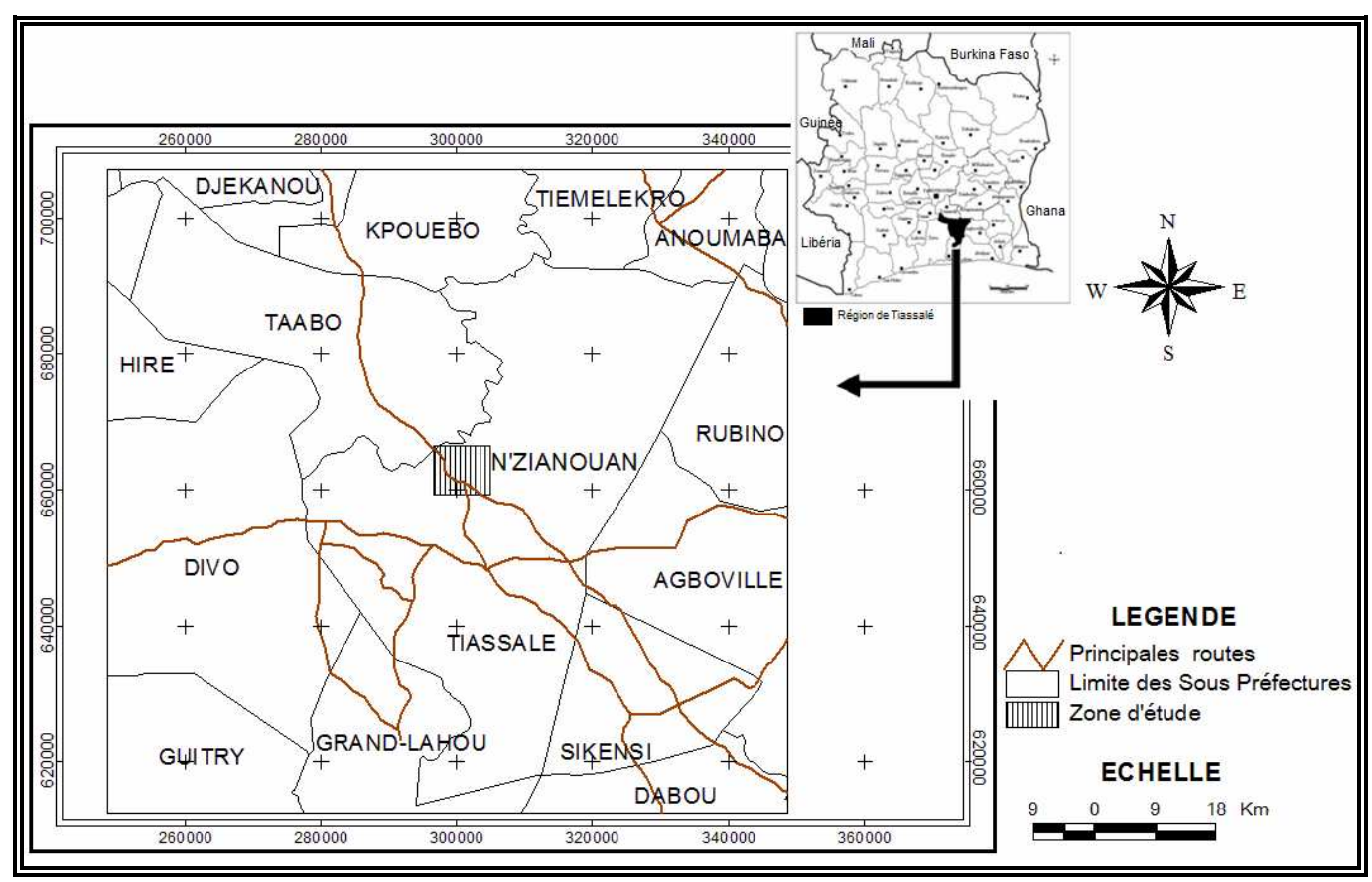

Figure 1: Localisation géographique de la zone d'étude.

\section{RÉSULTATS}

\section{Résultats hydrogéologiques}

Les résultats de la campagne piézométrique sont consignés dans le Tableau 1. Les profondeurs des nappes varient de 0,48 $\mathrm{m}$ à 4,52 $\mathrm{m}$, avec une moyenne de 1,24 $\mathrm{m}$, ce qui traduit que les nappes de la région sont peu profondes (Tableau 1).

Les zones les plus exposées à la pollution sont celles situées dans le bas-fond à proximité du lit de la rivière N'zi. Dans les puits qui captent ces nappes, la lame d'eau mesurée varie de $0,22 \mathrm{~m}$ à $2,91 \mathrm{~m}$, avec une moyenne de 1,69 m. Du fait de leur faible profondeur, ces eaux souterraines ne sont pas protégées contre les éventuelles sources de pollution.

\section{Résultats de l'étude hydro chimique Résultats des mesures in situ}

Les paramètres physiques des eaux souterraines et des eaux de la rivière N'zi mesurés sur le terrain sont répertoriés dans le Tableau 2. L'analyse de ce tableau montre que le $\mathrm{pH}$ des eaux de la rivière N'zi varie de 6,75 à 6,43; avec une moyenne de 6,61. L'eau de puits a un $\mathrm{pH}$ de 5,85 et l'eau du forage un $\mathrm{pH}$ de 7,05. Les eaux les plus minéralisées sont les eaux souterraines (eau du forage, avec $2487 \mu \mathrm{S} / \mathrm{cm}$ de conductivité). Les eaux de surface sont plus riches en matières en suspensions (16 $\mathrm{mg} / \mathrm{L})$ que les eaux souterraines $(4,2 \mathrm{mg} / \mathrm{L})$. Les eaux présentent dans l'ensemble une bonne saturation en oxygène dissous. Cependant, les eaux de la rivière N'zi sont les plus oxygénées, avec une saturation en oxygène dissous de $96,2 \%$.

Les paramètres physiques de l'eau de la rivière augmentent progressivement de l'amont de la rivière vers l'aval de cette même rivière (Figure 8). Il s'agit particulièrement de la conductivité qui passe de 78,5 $\mu \mathrm{S} / \mathrm{cm}$ à l'amont à $128,1 \mu \mathrm{S} / \mathrm{cm}$ à l'aval.

Résultats des analyses chimiques des ions et sels nutritifs

Les résultats des analyses chimiques réalisées sur les eaux (eaux souterraines et des eaux de la rivière N'zi) sont consignés dans le Tableau 3 . 
Les résultats montrent que l'eau du forage, avec des teneurs de 165,19 $\mathrm{mg} / \mathrm{L}$ en $\mathrm{HCO}_{3}^{-}$et $230 \mathrm{mg} / \mathrm{L}$ en $\mathrm{Cl}^{-}$est la plus minéralisée. L'eau de la rivière N'zi est la moins minéralisée. La classification hydrochimique des eaux à l'aide du diagramme triangulaire de Piper est donnée la Figure 9. Les eaux se classent en deux grands groupes. Les eaux bicarbonatées calciques sont observées au niveau de la rivière N'zi et les eaux chlorurées sodi-potassiques au niveau des eaux souterraines. Cela montre que dans les eaux de surface les ions $\mathrm{HCO}_{3}^{-}$sont les plus importants au niveau des anions et les ions $\mathrm{Ca}^{2+}$ sont les plus importants parmi les cations. Au niveau des eaux souterraines, les ions $\mathrm{Cl}^{-}$constituent les plus importants parmi les anions.

Résultats des analyses des Eléments Traces Métalliques (ETM)

Les résultats des analyses des ETM sont présentés dans le Tableau 4. Dans ce tableau, on note la présence de certains Eléments Traces Métalliques tels que : l'arsenic (As), le plomb $(\mathrm{Pb})$, le chrome $(\mathrm{Cr})$ et le cuivre $(\mathrm{Cu})$. La Figure 10 montre que l'eau de puits est plus riche en Chrome, avec une teneur de $0,421 \mathrm{mg} / \mathrm{L}$, ensuite vient l'eau de forage $(0,354 \mathrm{mg} / \mathrm{L})$ et l'eau de la rivière N'zi $(0,308$ $\mathrm{mg} / \mathrm{L})$. Les teneurs les plus élevées en plomb sont rencontrées dans l'eau de forage $(0,204$ $\mathrm{mg} / \mathrm{L})$ et l'eau de la rivière N'zi $(0,125 \mathrm{mg} / \mathrm{L})$.

\section{Résultats de l'étude statistique}

Le dendrogramme (Figure 11) issu de la Classification Hiérarchique Ascendante (CHA) a mis en évidence trois principaux regroupements des variables étudiées. Le premier regroupement qui traduit une origine naturelle de la minéralisation est défini par $\mathrm{T}^{\circ}$, $\mathrm{pH}, \quad \mathrm{O}_{2}, \quad \mathrm{SO}_{4}{ }^{2-}, \quad \mathrm{Na}^{+}$et $\mathrm{K}^{+}$. Le second regroupement qui est constitué de $\mathrm{Sal}$., $\mathrm{NO}_{3}^{-}$, $\mathrm{NO}_{2}^{-}, \mathrm{PO} 4, \mathrm{~Pb}, \mathrm{Cr}$ et $\mathrm{Cl}^{-}$, met en évidence une minéralisation liée aux apports des sels nutritifs d'origine anthropique. Le troisième regroupement est constitué de Mes, Turb., CE, $\mathrm{Ca}^{2+}, \mathrm{Mg}^{2+}, \mathrm{HCO}_{3}{ }^{-}$; $\mathrm{Eh}$; As et $\mathrm{Cu}$ témoigne du phénomène de pluviolessivage des sols.
Ces regroupements montrent que la minéralisation est liée à trois phénomènes importants. Le pluviolessivage qui concerne les sols pour les eaux de la rivière N'zi. Ensuite, la minéralisation à partir des sels nutritifs tels $\mathrm{NO}_{3}{ }^{-}, \mathrm{NO}_{2}{ }^{-}, \mathrm{PO}_{4}{ }^{3-}$ qui sont issus des activités anthropiques. Cette pollution concerne à la fois les eaux de nappes phréatiques et les eaux de surface. La minéralisation des eaux par le contact eauroche est également présente dans la zone mais à un degré moindre. Ce phénomène est observé au niveau des eaux souterraines.

La classification des points d'eau selon leur ressemblance pour le cadre d'un suivi est donnée par la Figure 12. Ce dendrogramme met en évidence deux grands regroupements des points d'eau étudiés. Le premier regroupement prend en compte les eaux de la rivière N'zi et le second contient les eaux souterraines. Dans le cadre d'un suivi de la qualité des eaux, il est important de choisir un point de suivi dans chaque regroupement. Ainsi deux points sont nécessaires pour le suivi.

\section{Résultats des analyses biologiques et microbiologiques des eaux}

Du point de vue microbiologique, les résultats des analyses indiquent la présence des coliformes fécaux principalement l'Echérichia coli dans la plupart des eaux étudiées (Tableau 5).

Les analyses révèlent également la présence de streptocoques fécaux dans l'ensemble des eaux de la zone d'étude à des taux élevés, avec respectivement 281820, 58200 et 24500 streptocoques fécaux pour $100 \mathrm{ml}$ d'eau.

Les Clostridiums Perfringens s'observent également dans toutes les eaux. $\mathrm{Au}$ niveau des eaux de la rivière $\mathrm{N}$ 'zi, on compte entre 51 et 75 Clostridiums Perfringens/20 ml d'eau. Pour les eaux de puits, on a 33 et 65 Clostridiums Perfringens/20 $\mathrm{ml}$ d'eau pour les eaux de forage.

Les coliformes Thermo tolérants sont les plus importants dans le milieu, avec des 
quantités de 24600 et de 112700 pour les eaux du site. On dénombre respectivement 24600 , 112700 et 19550 coliformes Thermo tolérants/100 ml pour les eaux de puits, de forage et de la rivière N'zi.

$\mathrm{La} \mathrm{DBO}_{5}$ et la $\mathrm{DCO}$ mesurées varient de 20 à $110 \mathrm{mg} / \mathrm{L}$ pour la $\mathrm{DBO}_{5}$ et de 54 à 233 $\mathrm{mg} / \mathrm{L}$ pour la DCO. La plus forte valeur de la DCO (233 mg/L) s'observe au niveau de l'eau de la rivière N'zi et la plus faible $54 \mathrm{mg} / \mathrm{L}$ dans l'eau de puits. Quant à la $\mathrm{DBO}_{5}$, la plus forte valeur est obtenue au niveau de l'eau de la rivière N'zi $(110 \mathrm{mg} / \mathrm{L})$ et la plus faible valeur au niveau de l'eau de puits $(20 \mathrm{mg} / \mathrm{L})$.

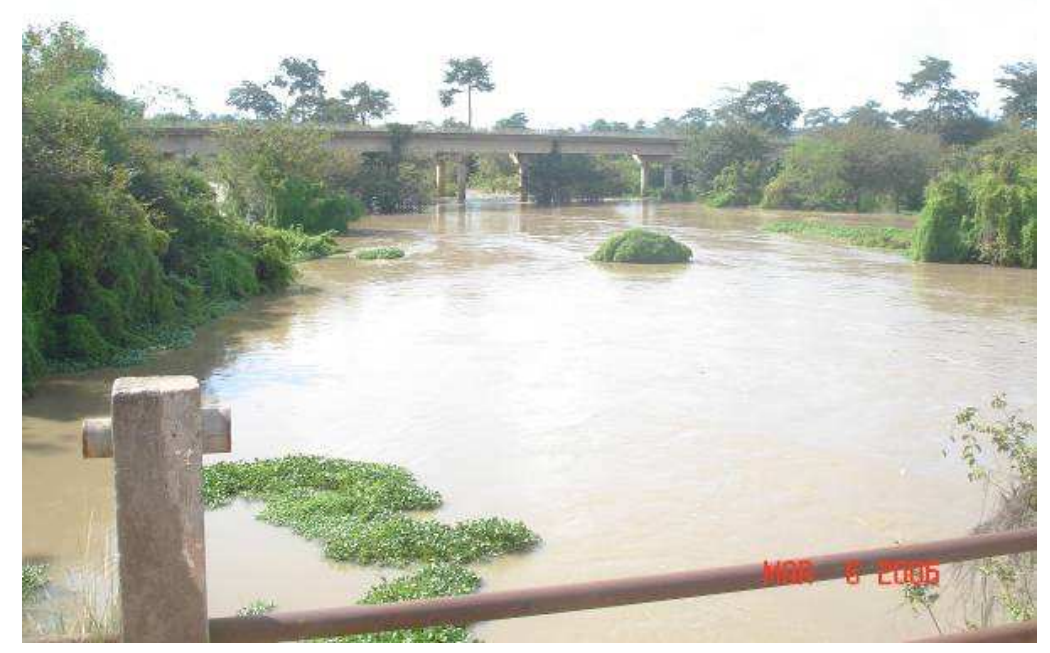

A

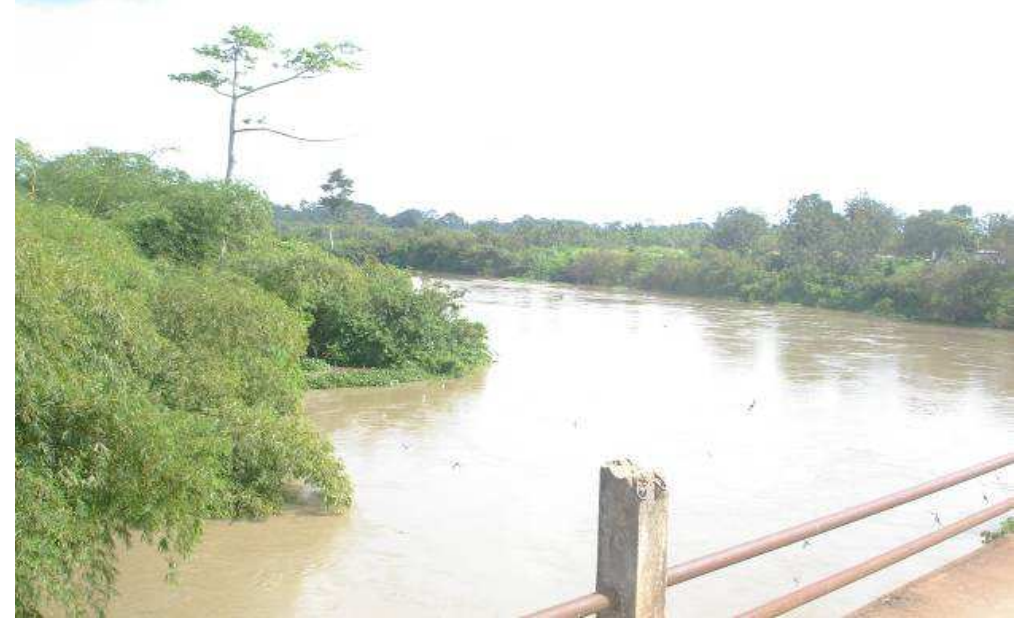

B

Figure 2 : Aperçu d'une importante crue de la rivière N'zi à N'zianouan pendant la période du 22 octobre 2010 . 


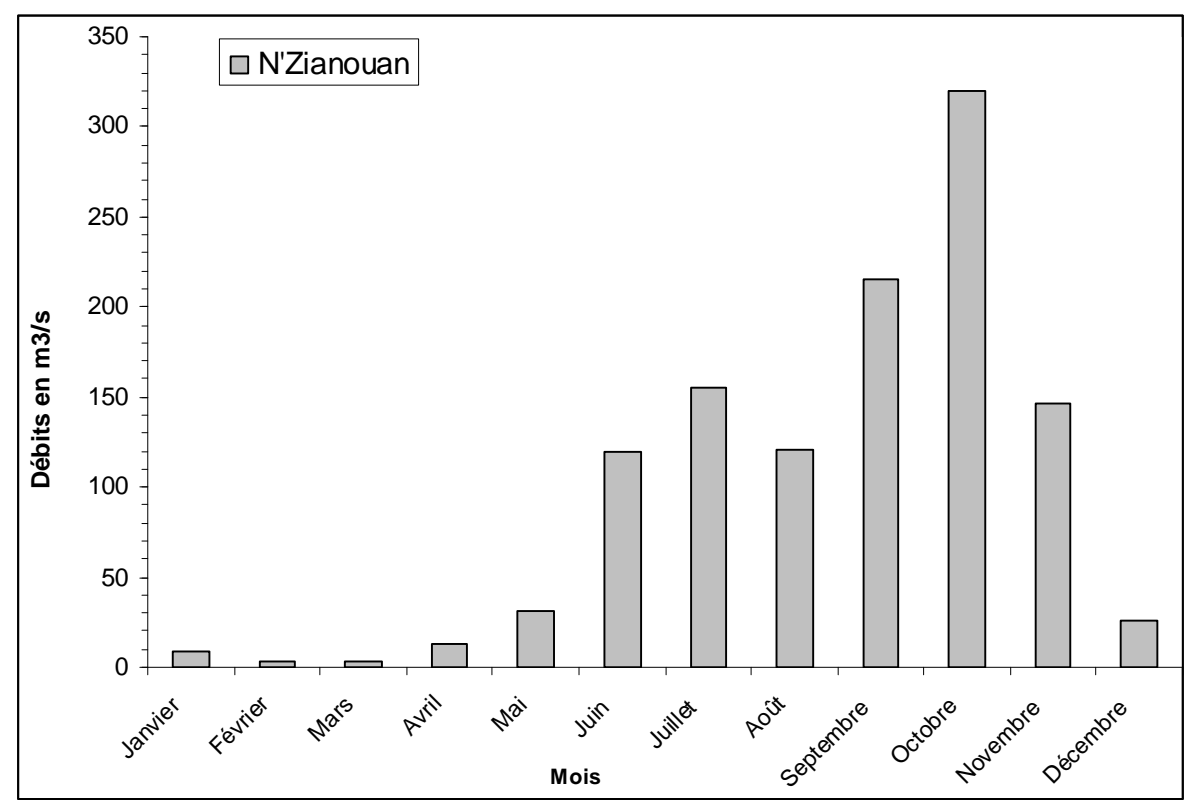

Figure 3 : Débits du N'zi à N'zianouan en 1968.

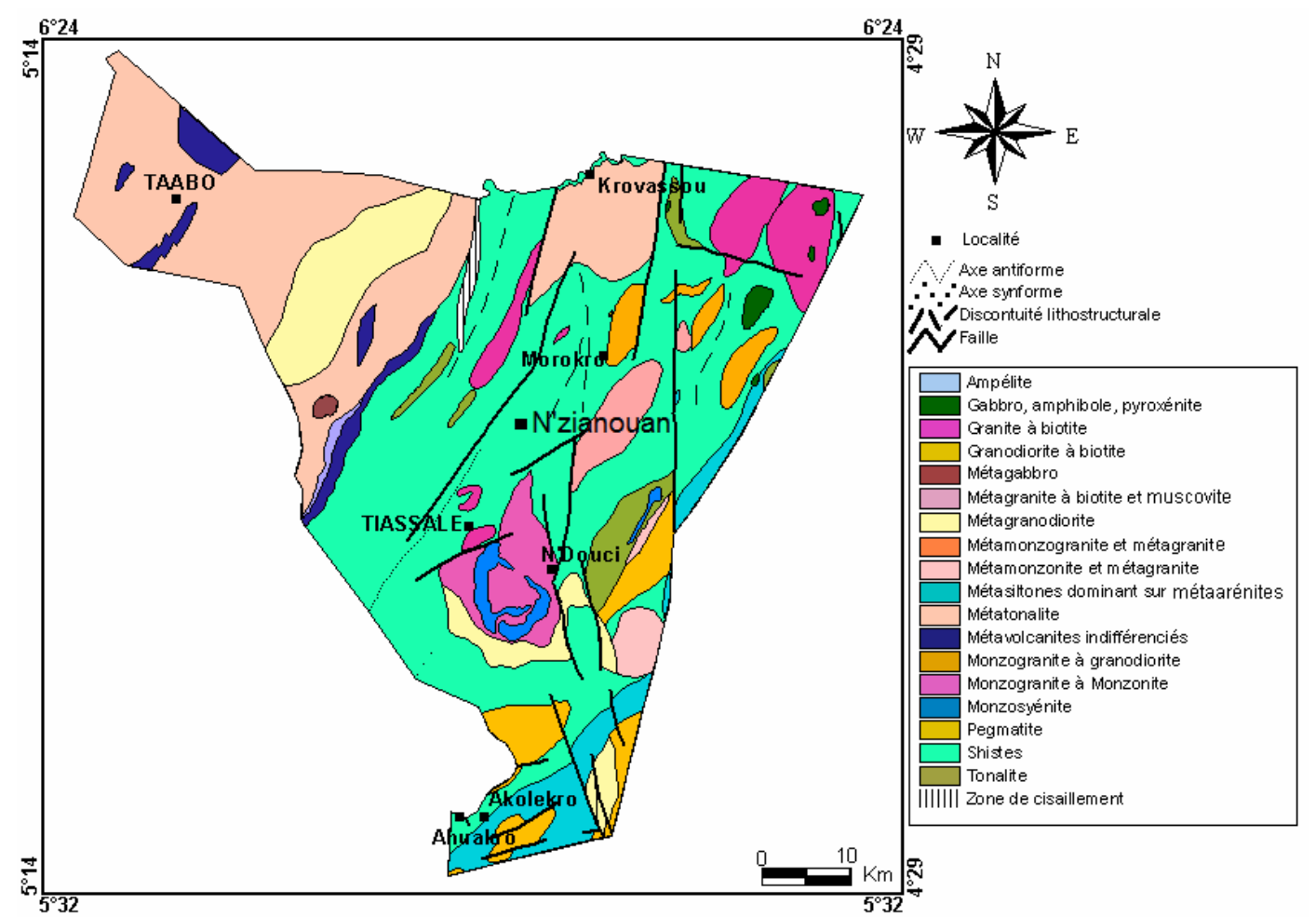

Figure 4 : Carte géologique du département de Tiassalé (Djaa, 2010). 


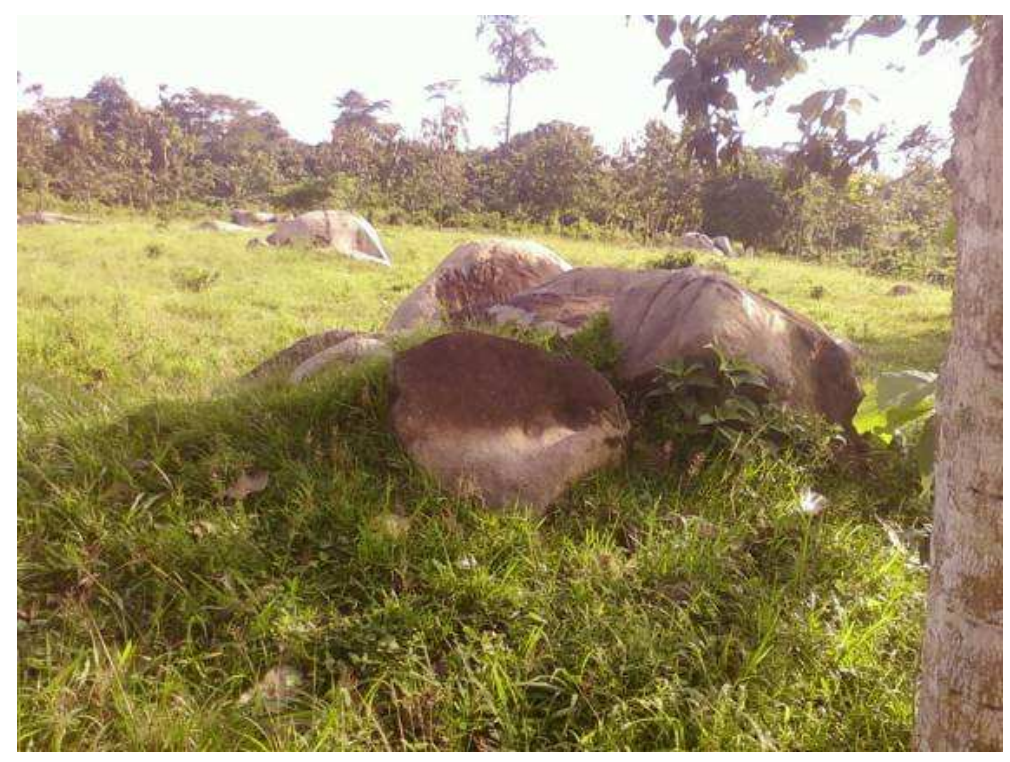

A

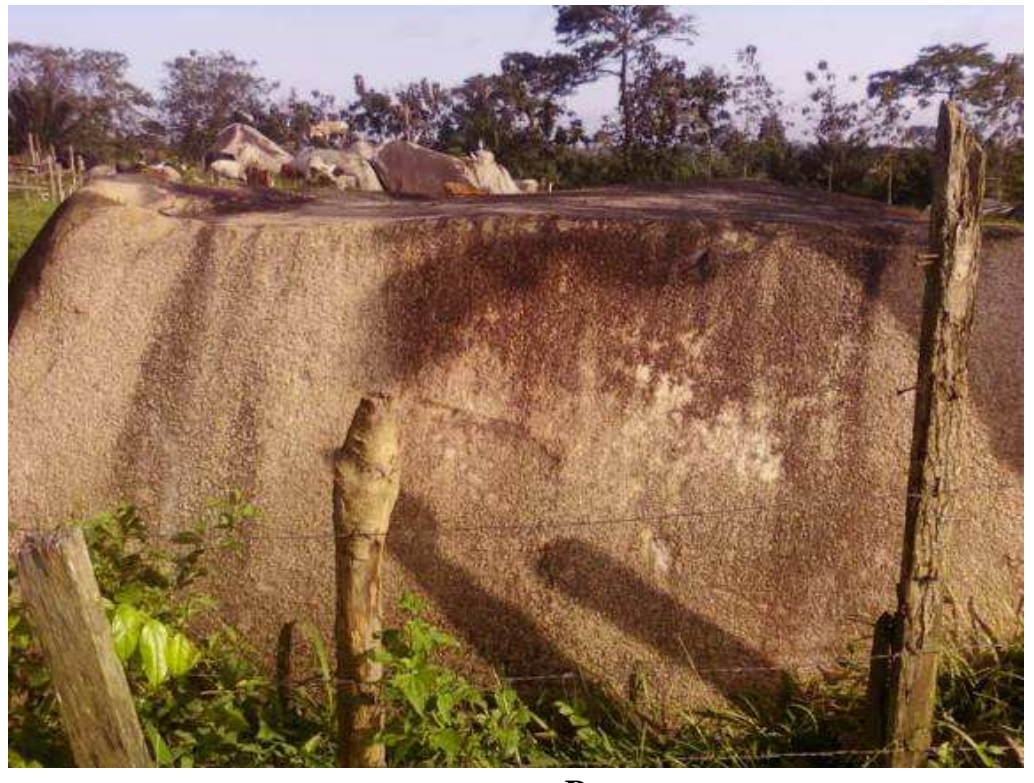

B

Figure 5 : Aperçu de quelques Chaos granitiques à la sortie de N'Douci dans le sens de Tiassalé. 


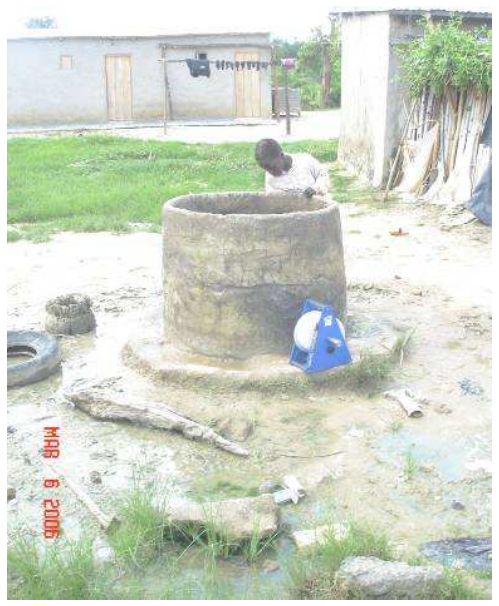

A

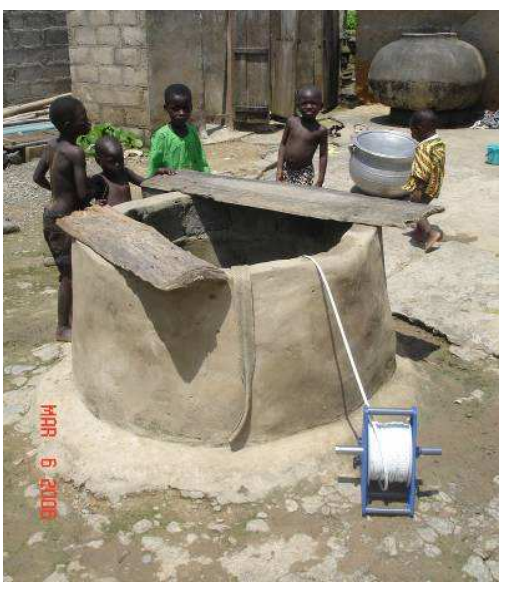

B

Figure 6: Puits villageois d'approvisionnement en eau potable de N'zianouan.

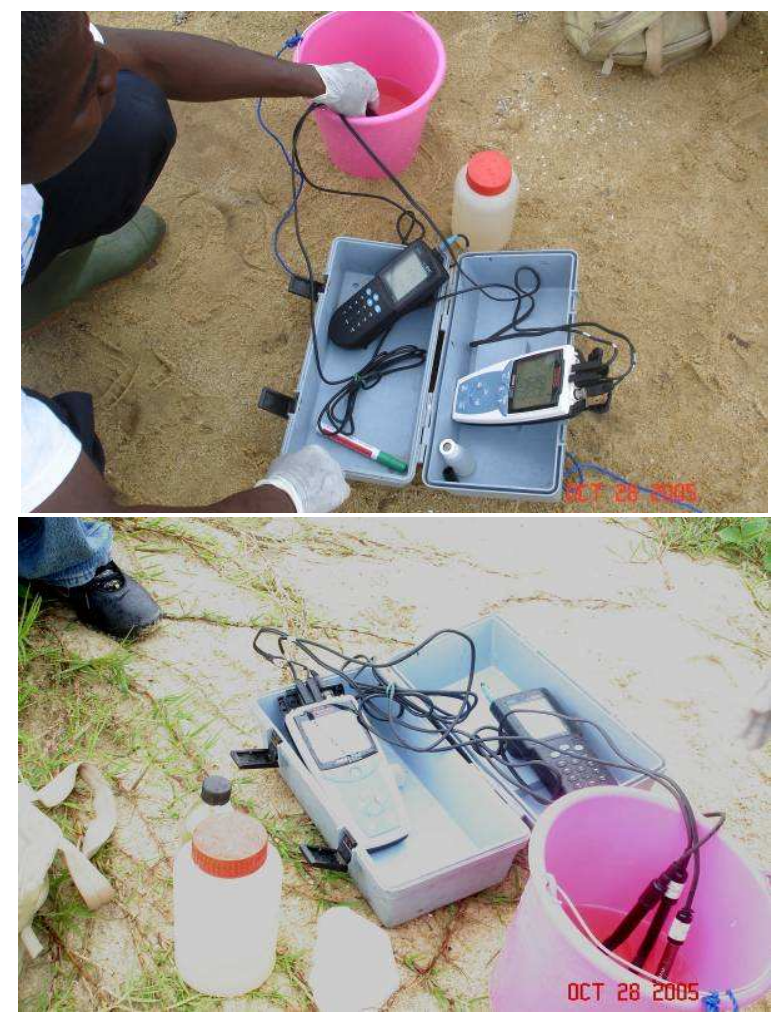

Figure 7 : Mesure in situ des paramètres physiques des eaux de la zone d'étude. 


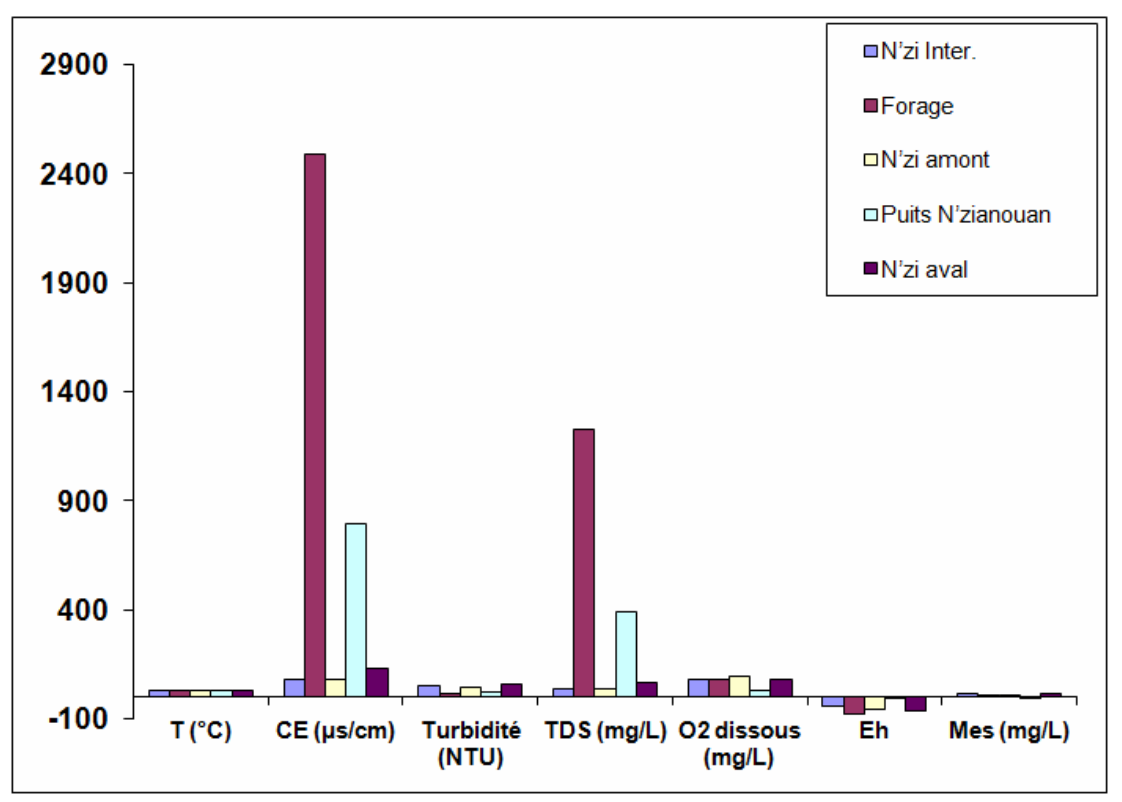

Figure 8: Évolution des paramètres physiques de l'eau de N'zianouan.

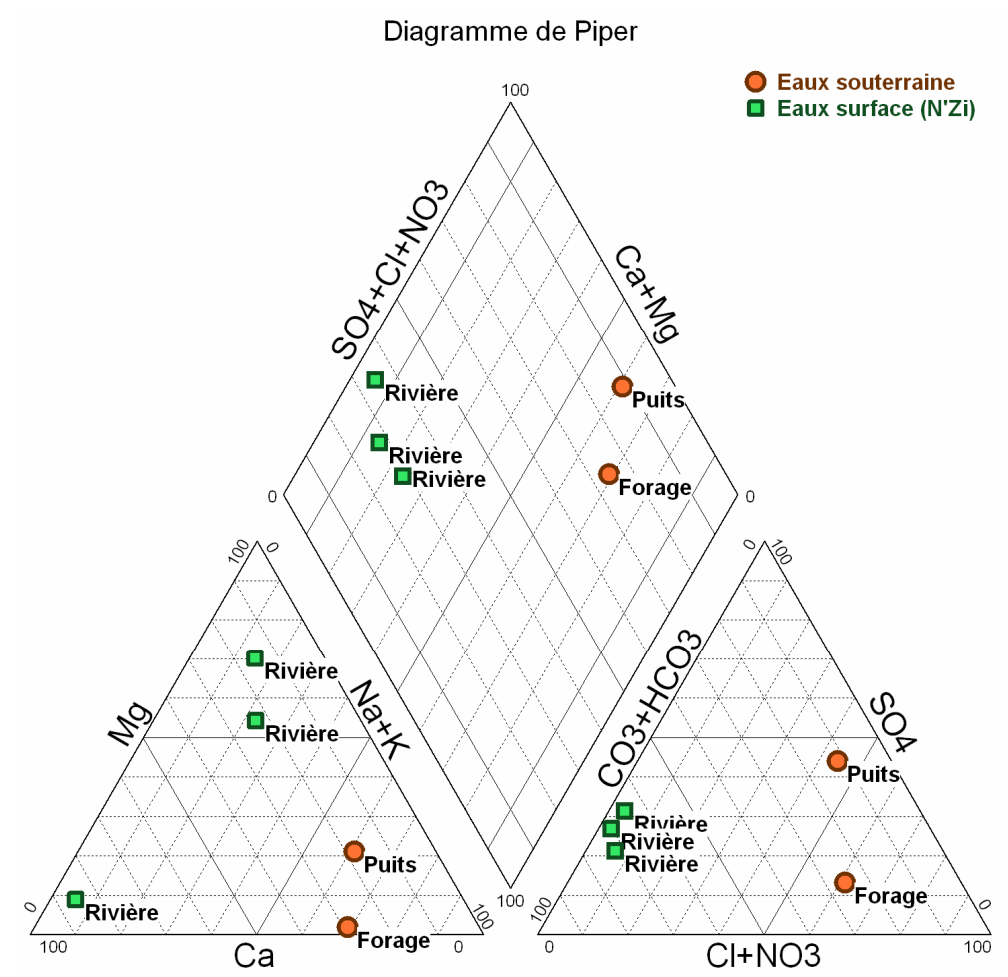

Figure 9 : Classification hydrochimique des eaux de N'zianouan. 


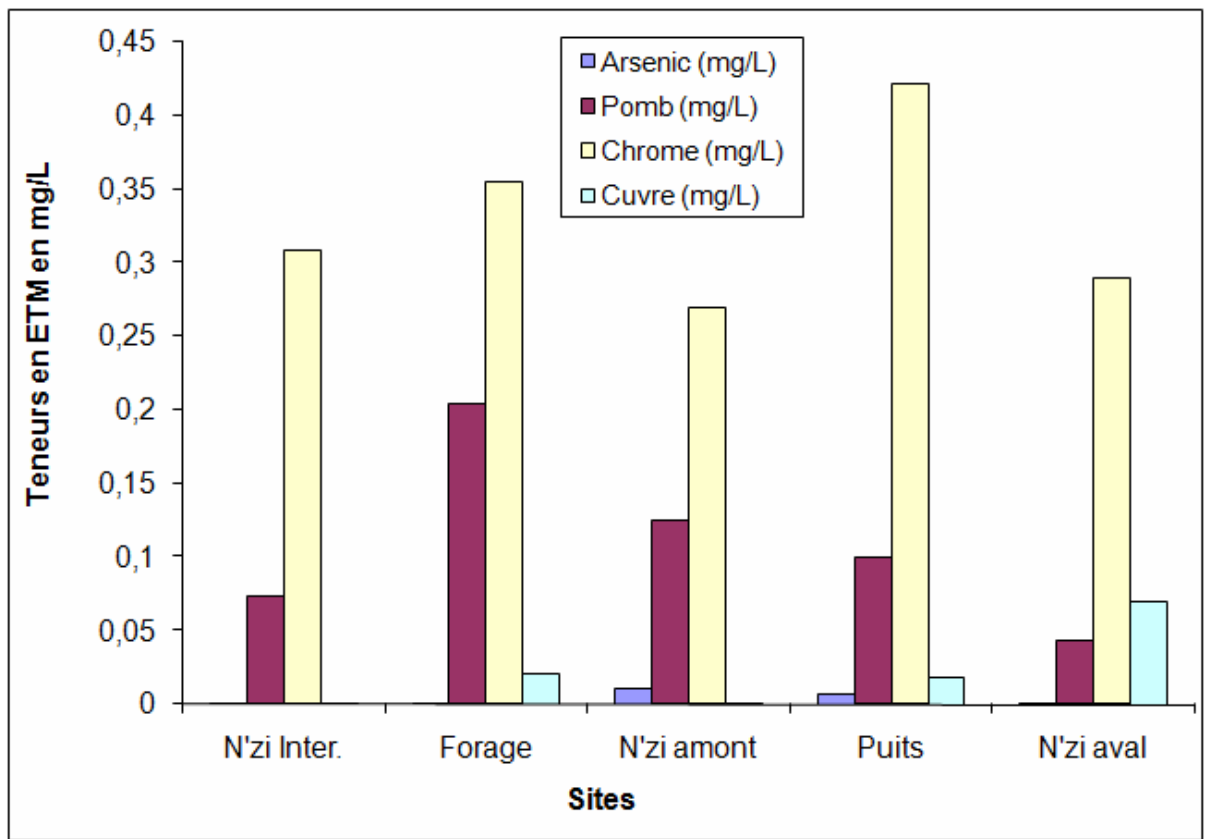

Figure 10 : Teneurs en Eléments Traces Métalliques dans les eaux de N'zianouan.

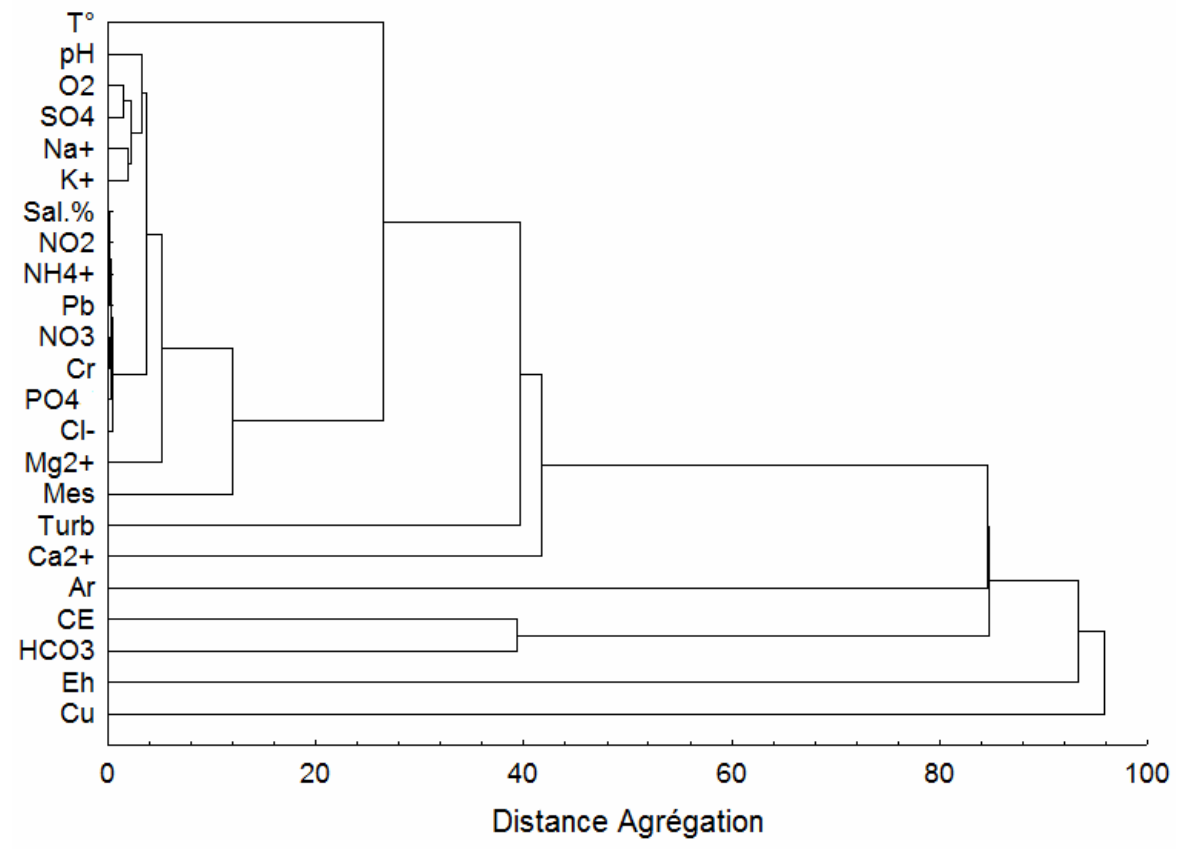

Figure 11 : Dendrogramme des paramètres physico-chimiques des eaux de N'zianouan. 


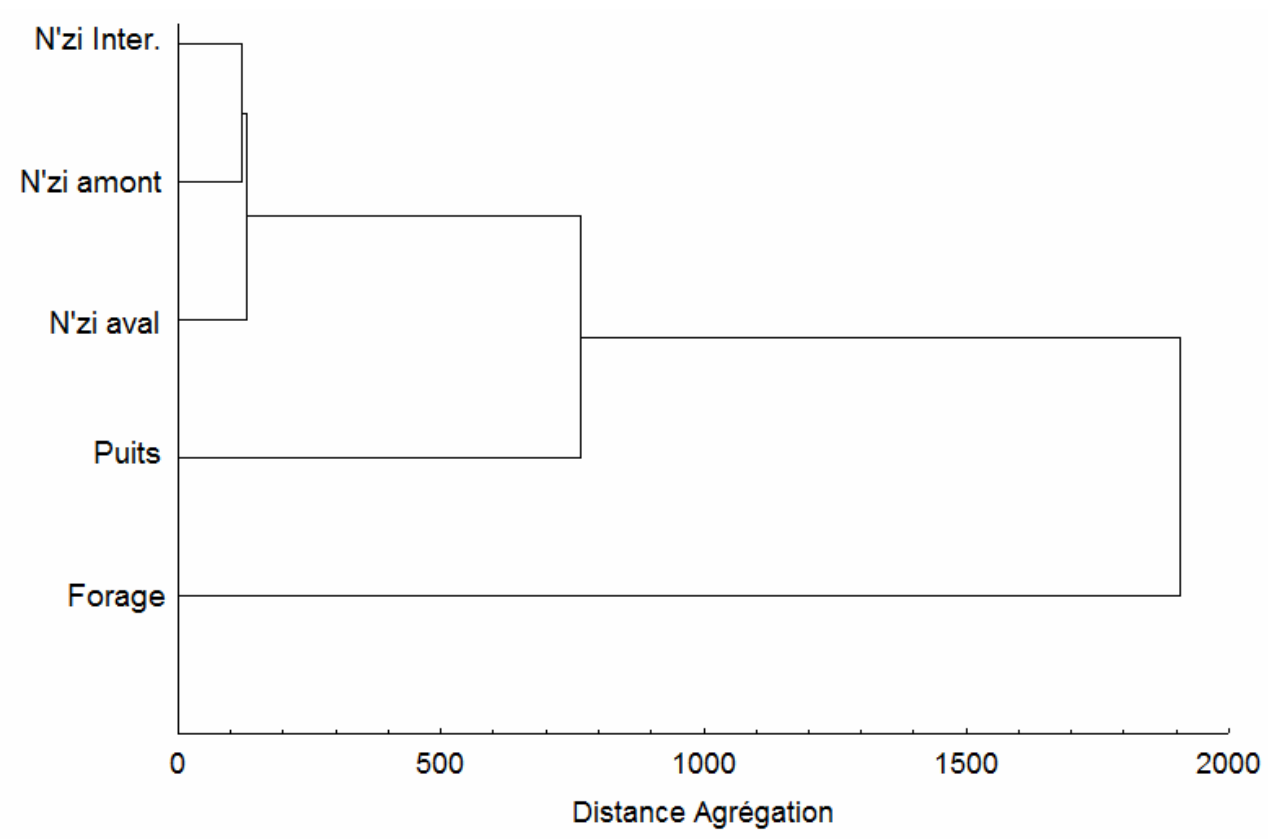

Figure 12 : Dendrogramme pour la classification des points d'eau de N'zianouan.

Tableau 1: Résultats de la compagne piézométrique du 22 octobre 2010.

\begin{tabular}{lcccccc}
\hline $\mathbf{N}^{\circ}$ & $\begin{array}{c}\text { Points de } \\
\text { mesure }\end{array}$ & $\mathbf{X}(\mathbf{m})$ & $\mathbf{Y}(\mathbf{m})$ & $\begin{array}{c}\text { Niveau } \\
\text { Piézométrique } \\
(\mathbf{m})\end{array}$ & $\begin{array}{c}\text { Côte de } \\
\text { l'eau (m) }\end{array}$ & $\begin{array}{c}\text { Lame } \\
\text { d'eau (m) }\end{array}$ \\
\hline $\mathbf{1}$ & P1 & 298405 & 664093 & 0,49 & 47,51 & 2,15 \\
$\mathbf{2}$ & P2 & 298449 & 664072 & 2,12 & 45,88 & 0,79 \\
$\mathbf{3}$ & P3 & 298540 & 664041 & 1,59 & 46,41 & 1,81 \\
$\mathbf{4}$ & P4 & 298557 & 663994 & 0,51 & 47,49 & 1,89 \\
$\mathbf{5}$ & P5 & 298560 & 664106 & 0,80 & 47,20 & 2,03 \\
$\mathbf{6}$ & P6 & 298568 & 664167 & 0,83 & 47,17 & 2,91 \\
$\mathbf{7}$ & P7 & 298572 & 664199 & 0,48 & 47,52 & 2,13 \\
$\mathbf{8}$ & P8 & 298582 & 664251 & 0,80 & 47,20 & 1,77 \\
$\mathbf{9}$ & P9 & 298590 & 664321 & 1,12 & 47,88 & 1,00 \\
$\mathbf{1 0}$ & P10 & 298604 & 664383 & 1,79 & 50,21 & 2,50 \\
$\mathbf{1 1}$ & P11 & 298526 & 664433 & 1,03 & 50,97 & 1,13 \\
$\mathbf{1 2}$ & P12 & 298429 & 664436 & 0,73 & 51,27 & 1,01 \\
$\mathbf{1 3}$ & P13 & 298301 & 664409 & 4,52 & 47,48 & 0,22 \\
$\mathbf{1 4}$ & P14 & 298406 & 664278 & 0,51 & 51,49 & 2,25 \\
Moyenne & & & & 1,24 & 48,26 & 1,69 \\
Min & & & & 0,48 & 45,88 & 0,22 \\
Max & & & & 4,52 & 51,49 & 2,91 \\
\hline
\end{tabular}


Tableau 2: Récapitulatif des mesures in situ des paramètres physiques des eaux.

\begin{tabular}{|c|c|c|c|c|c|c|c|c|c|c|c|c|}
\hline $\mathbf{N}^{\circ}$ & $\begin{array}{l}\text { Points de } \\
\text { mesures }\end{array}$ & Libelle & $\begin{array}{c}\text { Température } \\
\left({ }^{\circ} \mathbf{C}\right)\end{array}$ & pH & $\begin{array}{c}\text { Conductivité } \\
(\mu \mathrm{s} / \mathrm{cm})\end{array}$ & $\begin{array}{c}\text { Turbidité } \\
\text { (NTU) }\end{array}$ & $\begin{array}{c}\text { TDS } \\
(\mathrm{mg} / \mathrm{L})\end{array}$ & $\begin{array}{c}\mathrm{O}_{2} \text { dissous } \\
(\mathrm{mg} / \mathrm{L})\end{array}$ & $\begin{array}{c}\mathrm{O}_{2} \text { dissous } \\
(\%)\end{array}$ & $\begin{array}{c}\text { Eh } \\
(\mathbf{m v})\end{array}$ & $\begin{array}{l}\text { Salinité } \\
\text { (mg/L) }\end{array}$ & $\begin{array}{c}\text { Matière en } \\
\text { suspension } \\
(\mathrm{mg} / \mathrm{L})\end{array}$ \\
\hline & & Eau de la rivière & & & & & & & & & & \\
\hline 1 & N'zi Inter. & N'zi & 27,8 & 6,4 & 81,8 & 48 & 40 & 4,5 & $79,40 \%$ & $-41,1$ & 0 & 16 \\
\hline 2 & Forage & $\begin{array}{l}\text { Eau de forage } \\
\text { Eau de la rivière }\end{array}$ & 30 & 7,05 & 2487 & 13 & 1228 & 4,16 & $77,20 \%$ & $-80,1$ & 1,3 & 6 \\
\hline 3 & N'zi amont & N'zi & 29 & 6,67 & 78,5 & 47 & 38 & 5,34 & $96,20 \%$ & $-57,4$ & 0 & 11,2 \\
\hline 4 & Puits & $\begin{array}{l}\text { Eau de puits } \\
\text { Eau de la rivière }\end{array}$ & 27,6 & 5,82 & 791 & 22 & 387 & 1,7 & $29,90 \%$ & $-5,9$ & 0,4 & 4,2 \\
\hline 5 & N'zi aval & N'zi & 28 & 6,75 & 128,1 & 57 & 63 & 4 & $79,90 \%$ & $-61,1$ & 0,1 & 12,4 \\
\hline \multicolumn{2}{|c|}{ Moyenne } & & 28,48 & 6,538 & 713,28 & 37,4 & 351,2 & 3,94 & 0,7252 & $-49,12$ & 0,36 & 9,96 \\
\hline \multicolumn{2}{|c|}{ Max } & & 30 & 7,05 & 2487 & 57 & 1228 & 5,34 & $96,2 \%$ & $-5,9$ & 1,3 & 16 \\
\hline \multicolumn{2}{|c|}{ Min } & & 27,6 & 5,82 & 78,5 & 13 & 38 & 1,7 & $29,9 \%$ & $-80,1$ & 0 & 4,2 \\
\hline \multicolumn{2}{|c|}{ Ecart type } & & 1,01 & 0,46 & 1036,37 & 18,85 & 511,87 & 1,35 & $25 \%$ & 27,86 & 0,55 & 4,82 \\
\hline
\end{tabular}


Tableau 3: Résultats des analyses chimiques des eaux de Nzianouan.

\begin{tabular}{llccccc}
\hline $\mathbf{N}^{\circ}$ ordre & Paramètres chimiques & N'zi Inter. & Forage & N'zi amont & Puits & N'zi aval \\
\hline & IONS MAJEURS & & & & & \\
1 & Sodium $\left(\mathrm{Na}^{+}\right)(\mathrm{mg} / \mathrm{L})$ & 2,38 & 175,2 & 2,4 & 101,2 & 2,72 \\
2 & Potassium $\left(\mathrm{K}^{+}\right)(\mathrm{mg} / \mathrm{L})$ & 3,17 & 2,18 & 4,16 & 50,12 & 2,71 \\
3 & Calcium $\left(\mathrm{Ca}^{2+}\right)(\mathrm{mg} / \mathrm{L})$ & 56,23 & 64,12 & 4,32 & 33,45 & 3,99 \\
4 & Magnésium $\left(\mathrm{Mg}^{2+}\right)(\mathrm{mg} / \mathrm{L})$ & 3,56 & 2,67 & 6,12 & 23,75 & 11,07 \\
5 & Chlorures $\left(\mathrm{Cl}^{-}\right)(\mathrm{mg} / \mathrm{L})$ & 0,25 & 230 & 0,22 & 75,6 & 0,75 \\
6 & Sulfates $\left(\mathrm{SO}_{4}{ }^{2-}\right)(\mathrm{mg} / \mathrm{L})$ & 6 & 67 & 5 & 102 & 4 \\
7 & Bicarbonates $\left(\mathrm{HCO}_{3}^{-}\right)(\mathrm{mg} / \mathrm{L})$ & 15,86 & 165,19 & 16,59 & 34,16 & 17,32 \\
& LES SELS NUTRITIFS & & & & 0,34 & 0,26 \\
8 & Nitrates $\left(\mathrm{NO}_{3}{ }^{-}\right)(\mathrm{mg} / \mathrm{L})$ & 0,43 & 0,17 & 0,34 & 0,03 & 0,04 \\
9 & Nitrites $\left(\mathrm{NO}_{2}^{-}\right)(\mathrm{mg} / \mathrm{L})$ & 0,01 & 0,02 & 0,04 & 0,03 & 0,04 \\
10 & Ammonium $\left(\mathrm{NH}_{4}^{+}\right)(\mathrm{mg} / \mathrm{L})$ & 0,11 & 0,02 & 0,04 & 0,03 & 0,35 \\
11 & Phosphates $\left(\mathrm{PO}_{4}{ }^{3-}\right)(\mathrm{mg} / \mathrm{L})$ & 0,07 & 0,36 & 0,35 & 0,35 & 0,41 \\
\hline
\end{tabular}


Tableau 4: Résultats de l'analyse des Eléments Traces Métalliques (ETM) des eaux en mg/L.

\begin{tabular}{lccccccccc}
\hline Paramètres & N'zi Inter. & Forage & N'zi amont & Puits & N'zi aval & Moyenne & Min & Max & Ecart type \\
\hline Arsenic (mg/L) & $<0,001$ & $<0,001$ & 0,011 & 0,007 & $<0,001$ & 0,009 & 0,007 & 0,011 & 0,003 \\
Pomb (mg/L) & 0,073 & 0,204 & 0,125 & 0,099 & 0,043 & 0,109 & 0,043 & 0,204 & 0,061 \\
Chrome (mg/L) & 0,308 & 0,354 & 0,269 & 0,421 & 0,289 & 0,328 & 0,269 & 0,421 & 0,061 \\
Cuvre (mg/L) & $<0,001$ & 0,021 & $<0,001$ & 0,018 & 0,07 & 0,036 & 0,018 & 0,070 & 0,029 \\
\hline
\end{tabular}

Tableau 5 : Résultats des analyses biologiques et microbiologiques des eaux de N'zianouan.

\begin{tabular}{|c|c|c|c|c|c|c|}
\hline $\mathbf{N}^{\circ}$ ordre & Paramètres & N'zi Inter. & Forage & N'zi amont & Puits & N'zi aval \\
\hline & Paramètres de pollution biologique & & & & & \\
\hline 1 & $\mathrm{DCO}(\mathrm{mg} / \mathrm{L})$ & 233 & 66 & 160 & 54 & 105 \\
\hline \multirow[t]{2}{*}{2} & $\mathrm{DBO}_{5}(\mathrm{mg} / \mathrm{L})$ & 110 & 30 & 70 & 20 & 50 \\
\hline & MICROBILOGIE & & & & & \\
\hline 1 & Echérichia coli/100ml & $<1$ & 12200 & 450 & 14500 & 15000 \\
\hline 2 & Streptocoques Fécaux/100ml & 500 & 24500 & 5000 & 58200 & 281820 \\
\hline 3 & Clostridiums Perfringens/20ml & 51 & 65 & 84 & 33 & 75 \\
\hline 4 & Coliformes Thermo tolérants $/ 100 \mathrm{ml}$ & $<1$ & 112700 & 3180 & 24600 & 19550 \\
\hline
\end{tabular}




\section{DISCUSSION}

Les analyses in situ réalisées sur les eaux de la région de N'zianouan montrent que la température moyenne des eaux qui est de $28,48{ }^{\circ} \mathrm{C}$ s'inscrit très bien dans l'intervalle de variation des températures des eaux en Côte d'Ivoire par comparaison avec les travaux d'Ahoussi et al. (2010 et 2011). Ces températures correspondent aux variations saisonnières des températures atmosphériques ambiantes. Selon Tandia et al. (1999), cela indique l'ouverture du système aquifère, donc de sa vulnérabilité vis-à-vis de la pollution. Les eaux les plus minéralisées sont les eaux souterraines, avec pour conductivité maximale $2487 \mu \mathrm{S} / \mathrm{cm}$. Les matières en suspensions sont plus importantes dans les eaux de surface (16 $\mathrm{mg} / \mathrm{L}$ pour l'eau de la rivière N'zi). Les eaux de la zone présentent une bonne saturation en oxygène dissous. Les eaux de la rivière N'zi sont les plus oxygénées, avec une saturation en oxygène dissous de $96,2 \%$.

Les eaux sont acides, avec un $\mathrm{pH}$ moyen de 6,61 pour les eaux de la rivière N'zi et de 6,45 pour les eaux souterraines. L'acidité des eaux constitue un des caractères essentiels des eaux en Côte d'Ivoire. En effet, selon Tapsoba (1995) et Martini et al. (2009), l'acidité des eaux en zone tropicale humide est principalement liée à la décomposition de la matière organique végétale, avec la production de $\mathrm{CO}_{2}$ dans les premières couches du sol d'après la réaction suivante :

$$
\stackrel{\mathrm{CH}_{2} \mathrm{O}+\mathrm{O}_{2}}{\rightleftarrows} \mathrm{H}^{+}+\mathrm{HCO}_{3}^{-} \text {(1) } \mathrm{CO}_{2}+\mathrm{H}_{2} \mathrm{O}
$$

Aussi, selon Faillat et Drogue (1993), la présence dans l'eau de $\mathrm{CO}_{2}$ provenant des sols facilite-t-elle l'hydrolyse des minéraux silicatés et la formation des ions $\mathrm{HCO}_{3}{ }^{-}$. C'est ce phénomène qui explique l'abondance des bicarbonates dans les eaux de la région.

Les eaux de la région contiennent les coliformes fécaux du type Eschérichia coli. Cela montre que les eaux sont soumises une pollution bactériologique récente. En effet, la commune de N'zianouan étant dépourvue d'un système d'assainissement adéquat. Les eaux de surface sont utilisées par les populations comme des collecteurs d'ordures ménagères et des déchets de toutes sortes. Ainsi Boutin (1993) explique la présence des coliformes fécaux et des streptocoques fécaux dans les eaux de puits par une contamination d'origine fécale et donc la possibilité que les germes pathogènes dangereux soient présents dans l'eau. Les streptocoques et coliformes fécaux sont des indicateurs d'une pollution fécale et sont en grande partie d'origine humaine. La présence de coliformes fécaux confirme l'influence des eaux de N'zianouan par les activités anthropiques. Aussi l'origine fécale de la pollution des ressources en eau de la Côte d'Ivoire a-t-elle été mise en évidence par plusieurs auteurs tels que Claon (1997) dans les eaux souterraines des communes de Koumassi et de Port-Bouët et Ahoussi et al. (2009) au niveau des eaux souterraines de la ville d'Abidjan. À cette pollution bactériologique s'ajoute une pollution métallique marquée par la présence des Eléments Traces Métalliques dans les eaux de la rivière N'zi. Les eaux de la zone d'étude sont soumises à une influence anthropique.

\section{Conclusion}

Les travaux réalisés dans la commune de N'zianouan ont permis une caractérisation hydrogéochimique et microbiologique des ressources en eau. Dans la zone d'étude, la profondeur des nappes phréatiques varie de $0,48 \mathrm{~m}$ à 4,52 $\mathrm{m}$, avec une moyenne de 1,24 $\mathrm{m}$. Ces eaux sont peu profondes et sont très vulnérables à la pollution d'origine anthropique. Les analyses physico-chimiques des eaux montrent qu'elles sont moyennement minéralisées pour les eaux souterraines, avec maximum de $2487 \mu \mathrm{S} / \mathrm{cm}$ et faiblement pour les eaux de la rivière N'zi $(121,9 \mu \mathrm{S} / \mathrm{cm})$. Les eaux du site se classent en deux grands groupes. Les eaux de la rivière N'zi sont bicarbonatées calciques et les eaux souterraines (eau de forage et eau de puits) sont chlorurées sodi-potassiques. Dans ces eaux, on note la présence des Eléments Traces Métalliques (As, $\mathrm{Zn}, \mathrm{Cr}$ et $\mathrm{Pb}$ ) à des teneurs élevées. Les analyses microbiologiques montrent également la présence de coliformes fécaux (Echérichia coli), de coliformes 
Thermo tolérants, de streptocoques fécaux et de Clostridiums Perfringens dans les eaux (eau de la rivière N'zi, de puits et de forage) $\mathrm{du}$ site du projet. La présence de ces microorganismes et des Eléments Traces Métalliques dans les eaux de la zone de N'zianouan montre que celles-ci sont exposées à une pression anthropique qui détériore gravement leur qualité physicochimique et bactériologique.

\section{REFERENCES}

Ahoussi KE, Soro N, Soro G, Lasm T, Oga MS, Zadé S. 2008. Groundwater Pollution in Africans Biggest Towns: Case of the Town of Abidjan (Côte d'Ivoire). European Journal of Scientific Research, 20(2): 302-316.

Ahoussi KE, Soro N, Soro G, Oga MS, Zadé S. 2009. Caractérisation de la qualité physico-chimique et bactériologique des eaux de puits de la ville d'Abidjan (Côte d'Ivoire). Africa Geoscience Review, 16(3): 199-211.

Ahoussi KE, Soro N, Koffi YB, Soro G, Biémi J. 2010. Origine de la minéralisation des eaux des aquifères discontinus sous couvert forestier de la zone Sud de la Côte d'Ivoire: cas de la région d'Abidjan - Agboville. International Journal of Biological and Chemical Sciences, 4(3): 782-797.

Ahoussi KE, Oga YMS, Koffi YB, Kouassi AM, Soro N, Biémi J. 2011. Caractérisation hydrogéochimique et microbiologique des ressources en eau du site d'un Centre d'Enfouissement Technique (CET) de Côte d'Ivoire : cas du CET de Kossihouen dans le District d'Abidjan (Côte d'Ivoire). International Journal of Biological and Chemical Sciences, 5(5): 2114-2132.

Beliard C, Casanova R. 1972. Excursion géologique à N'Douci ; DUES $1^{\text {ere }}$ année, Université d'Abidjan, département, de géologie, Série doc, $\mathrm{N}^{\circ}$ 9, $25 \mathrm{p}$.

Biémi J. 1992. Contribution à l'étude géologique, hydrogéologique et par télédétection des bassins versants
Subsahéliens du socle précambrien d'Afrique de l'Ouest : Hydrostructurale, hydrochimie et isotopie des aquifères discontinus des sillons et aires granitiques de la Haute Marahoué (Côte d'Ivoire). Thèse d'Etat, Université Nationale de Côte d'Ivoire, p. 480.

Boutin C. 1993. L'eau des nappes phréatiques superficielles, une richesse naturelle mais vulnérable. L'exemple des zones rurales du Maroc. Sciences de l'Eau, 6(3): 357365.

Claon JS. 1997. Consommation d'eau de puits dans quatre communes de la ville d'Abidjan desservies par le réseau de distribution d'eau potable. Thèse de Doctorat en pharmacie, Université d'Abidjan, Côte d'Ivoire, p. 197.

Coulibaly A, Mondé S, Wognin VA, Aka K. 2008. State of anthropic pollution in theestuary of Ebrié lagoon (Côte d'Ivoire) by analysis of the metal elements traces. European Journal of Scientific Research, 19(2): 372-390.

Djaa KA. 2010. Etude des fractures de productivité des forages de la région de Tiassalé (Sud de la Côte d'Ivoire). Mémoire de DEA, Université de Cocody Abidjan, p. 67

Delor C, Diaby I, Yao B, Tastet J-P, Vidal M, Chiron JC, Dommanget A. 1992. Notice explicative de la carte géologique de la Côte d'Ivoire au 1/200 000, feuille de Dimbokro, Mémoire de la géologie de Côte d'Ivoire, Abidjan, Côte d'Ivoire, 30 p.

Faillat JP, Drogue C. 1993. Différenciation hydrochimique de nappes superposées d'altérites et de fissures en socle granitique. Journal des Sciences Hydrologiques, 3(6): 215-229.

Güler C, Thyne GD, Mccray JE, Tuner AK. 2002. Evaluation of graphical and multivariate statistical methods for classification of water chemistry data. Hydrogeology Journal, 10: 455-474.

Kouzana L, Ben Mammou A, Gaaloul N. 2007. Intrusion marine et salinisation des eaux d'une nappe phréatique côtière 
(Korba, Cap-Bon, Tunisie). Geo-EcoTrop., 31: 57-70.

Matini L, Moutou JM, Kongo-Mantono MS. 2009. Evaluation hydrochimique des eaux souterraines en milieu urbain au SudOuest de Brazzaville, Congo. Afrique Science, 05(1): 82-98.

Oga MS, Lasm T, Yao TK, Soro N, Saley MB, Kouassi D, Gnamba F. 2009. Caractérisation chimique des eaux des aquifères de Fracture: Cas de La Région de Tiassalé en Côte d'Ivoire. European Journal of Scientific Research, 31(1):7287.

Tandia AA, Diop ES, Gaye CB. 1999. Pollution par les nitrates des nappes phréatiques sous environnement semiurbain non assaini : exemple de la nappe de Yeumbeul, Sénégal. Journal of African Earth Sciences, 29(4): 809-822.

Soro G, Métongo SB, SORO N, Ahoussi KE, Kouamé Koffi F, Zadé SGP, Soro T. 2009. Métaux lourds ( $\mathrm{Cu}, \mathrm{Cr}, \mathrm{Mn}$ et $\mathrm{Zn}$ ) dans les sédiments de surface d'une lagune tropicale africaine : cas de la lagune Ebrié (Côte d'Ivoire). International Journal of Biological and Chemical Sciences, 3(6): 1408-1427.

Soro N, Ouattara L, Dongo K, Kouadio KE, Ahoussi KE, Soro G, Oga YM-S, Savane
I, Biémi J. 2010. Déchets municipaux dans le District d'Abidjan en Côte d'Ivoire: sources potentielles de pollution des eaux souterraines. International Journal of Biological and Chemical Sciences, 4(6): 2203-2219.

Tagini B. 1971. Esquisse structurale de la Côte d'Ivoire, Essai de géotechnique régionale, Sodemi, Abidjan, 302 p.

Tapsoba S. A. 1995. Contribution à l'étude Géologique et Hydrogéologique de la région de Dabou (Sud de la Côte d'Ivoire): Hydrochimie, Isotopie et Indice de vieillissement des eaux souterraines, Thèse de $3^{\text {è }}$ cycle de l'Université Nationale de Côte d'Ivoire, p. 200.

Yermani M, Zouari K, Michelot JL, Mamou A, Moumni L. 2003. Approche géochimique du fonctionnement de la nappe profonde de Gafsa Nord (Tunisie centrale). Journal des Sciences Hydrologiques, 48(1): 95-108.

Yidana SM, Ophori D, Banoeng-Yakubo B. 2008. A multivariate statistical analysis of surface water chemistry data - The Ankobra Basin, Ghana. Journal of Environmental Management, 86: 80-87. 